\title{
Estrategias de Responsabilidad Social Empresarial en organizaciones del sector construcción en Medellín
}

\section{Strategies of Corporate Social Responsibility in the construction sector companies of Medellín}

\begin{abstract}
Dra. Yanyn Rincón Quintero es docente e investigadora en el Tecnológico de Antioquia-Institución Universitaria (Colombia)
\end{abstract} (yanyn.rincon@tdea.edu.co) (https://orcid.org/0000-0003-2427-3161)

Daniela Montoya Álvarez es Licenciada en Administración Financiera del Tecnológico de Antioquia-Institución Universitaria (Colombia). (danielamontoya511@gmail.com) (https://orcid.org/0000-0002-6648-6707)

Paola Vélez Patiño es Licenciada en Administración Financiera del Tecnológico de Antioquia-Institución Universitaria (Colombia) (paola23v@gmail.com) (https://orcid.org/0000-0003-2183-6600)

\section{Resumen}

La responsabilidad social sigue siendo tema de especial interés para organizaciones y empresas dada su correspondencia con los nuevos retos glocales. La presente investigación tiene como propósito describir las estrategias de Responsabilidad Social Empresarial empleadas por las empresas del sector construcción en Medellín. Se trata de una investigación desarrollada bajo el paradigma holístico integrado, de tipo descriptiva bajo un diseño contemporáneo de fuente mixta-transeccional descriptiva. Se realizó una revisión documental que posibilitó la conceptualización de la variable objeto de estudio al tiempo que se aplicó una escala estructurada con base en un cuestionario de 15 ítems, teniendo como unidades de información a los encargados de la función de Responsabilidad Social de las empresas del sector construcción inscritas en FENALCO Antioquia. Los hallazgos evidencian que la práctica de la Responsabilidad Social Empresarial en las organizaciones objeto de estudio es muy joven, pues evidencian menos de 10 años de práctica y está enfocada en estrategias orientadas a la comunidad y a los trabajadores. Se verifica también que aplican estrategias de Mercadeo Socialmente Responsable y usan fundamentalmente herramientas del medioambiente con lo que se vislumbra como una estrategia de Responsabilidad Social Empresarial de iniciación. Se recomienda la diversificación de estrategias de responsabilidad social en correspondencia con los factores e indicadores que proveen las diversas herramientas y mecanismos de Responsabilidad Social Empresarial.

\begin{abstract}
Social responsibility continues to be a topic of special interest for organizations and companies given its correspondence with new global challenges. The purpose of this research is to describe the corporate social responsibility strategies used by companies in the construction sector in Medellin. It is a research developed under the integrated holistic paradigm, under a contemporary cross-sectional descriptive design. A literature review was carried out making possible the conceptualization of the object of study. A structured scale based on a questionnaire of 15 items was applied, with information units corresponding to those in charge of the Social Responsibility function of the construction companies subscribed in FENALCO, Antioquia. The findings show that the practice of corporate social responsibility in the companies under study is very new, less than 10 years old. It is focused on strategies oriented towards the community and workers. The research verifies that socially responsible marketing strategies are also applied, using mainly tools related to the environment and which are envisioned as a form of corporate social responsibility in its initial stages. Recommendations include the diversification of social responsibility strategies in correspondence with the factors and indicators that provide the various tools and mechanisms of corporate social responsibility.
\end{abstract}

\section{Palabras clave I Keywords}

Responsabilidad social, empresa, organizaciones, estrategia, responsabilidad social empresarial Social Responsibility, company, organizations, strategy, corporate social responsibility

Cómo citar: Rincón Quintero, Y., Montoya Álvarez, D. \& Vélez Patiño, P. (2018). Estrategias de Responsabilidad Social Empresarial en organizaciones del sector construcción en Medellín. Retos Revista de Ciencias de la Administración y Economía, 8(16), 79-94. https://10.17163/ret.n16.2018.06 


\section{Introducción}

La Responsabilidad Social Empresarial (RSE) ha cobrado un papel clave en individuos y organizaciones en virtud de la necesidad de revalorizar las condiciones de vida y relacionamiento en el planeta. Las empresas a escala global han persistido en el desarrollo de formas idóneas de responsabilidad social que permitan mejores condiciones para la productividad y la competitividad, en correspondencia con los públicos organizacionales (stakeholders).

La responsabilidad es un concepto centenario según las consideraciones de Cardona (2017) y Rincón \& Ramírez (2018), enfatizándose que su concepción ha tenido un boom desde inicios del siglo XXI, dada la vinculación con los grupos de interés en organizaciones públicas y privadas para orientar un cambio de paradigma inclusivo y de integración para el desarrollo sostenible de las economías.

La presente investigación tiene como propósito describir las estrategias de Responsabilidad Social Empresarial (RSE) empleadas por las empresas del sector construcción en Medellín (Colombia), para lo cual se identificó la orientación de estrategias de Responsabilidad Social Empresarial con los públicos organizacionales o grupos de interés (stakeholders). En este sentido se busca determinar las estrategias de responsabilidad social empleadas e identificar las herramientas de responsabilidad social en el marco de las empresas objeto de estudio.

En Colombia, el empleo de la responsabilidad social como una dimensión estratégica organizacional es cada vez más recurrente. Así lo manifiesta Pérez (2014) al señalar que Colombia supera a Chile y México en reportes de responsabilidad social. Según Rincón, Caridad \& Salazar (2017):

[...] la sociedad actual hace cada vez más recurrentes sus demandas y reclamos a las organizaciones para que actúen en función del triple bottom line, refiriéndose a los tres vectores: económico, social y ambiental, que condicionan la inserción en los mercados de manera competitiva sostenidamente en pro del desarrollo sostenible de cara a una economía del bienestar (p. 49).

Según Rincón y Ramírez (2018, p.98) "la responsabilidad social es un tema que ha cobrado impulso en las últimas décadas constituyéndose en una especie de (boom) su uso y aplicación en todo tipo de organizaciones". Las empresas del sector construcción no escapan a la necesidad de aportar el concepto de responsabilidad social, ya que estas dada su naturaleza articulan el componente social, económico y medioambiental en corcondancia con el triple botton line, haciendo necesario observar de qué manera las empresas del sector construcción estan aplicando responsabilidad social empresarial, el cual "si bien para muchos está de moda, orienta la capacidad de responder mediante la articulación de acciones coordinadas a las necesidades de un colectivo cada vez mejor informado, más organizado y dispuesto a lograr metas y objetivos comunes". Rincón (2017, párr.2).

El sector de la construcción en Colombia, es determinante en el crecimiento del PIB destacando por su generación de empleo, crecimiento económico, y diversificación (DANE, 2017). 


\section{Revisión de la literatura}

\subsection{Estado del arte}

A continuación se presentan algunas investigaciones recientes en torno a la Responsabilidad Social Empresarial, en lo posible específica al sector construcción o afines. Se presenta en primer lugar la investigación de Padilla, Arévalo, Bustamante \& Vidal (2017), quienes en su trabajo titulado «Responsabilidad social empresarial y desempeño financiero» analizan la relación entre prácticas de Responsabilidad Social Empresarial y el desempeño financiero de compañías pertenecientes al sector industrial del plástico. Este estudio hizo uso de la encuesta diseñada con escala de likert, como método de recolección de información para demostrar cómo pueden las empresas ser responsables socialmente. Para ello se seleccionó una muestra de 192 empresas de Quito (Ecuador).

Por su parte, Padilla et al. (2017), determinaron la existencia de correlaciones de variables de RSE con los índices financieros; además, observaron que las empresas ecuatorianas muestran un nivel medio de implementación del concepto de RSE. Esta correlación no muestra dependencias estadísticamente significativas entre el índice de RSE y el desempeño financiero. De acuerdo con los resultados de este estudio, sí existe una relación significativa entre el pilar de responsabilidad social y el rendimiento sobre las ventas o activos (ROS), y entre los pilares de responsabilidad económica y ambiental con respecto al retorno sobre el patrimonio (ROE).

Conforme a los referentes internacionales sobre el tema, Poussenkova, Nikitina, Rowe \& Fjaertoft (2016), en su investigación «Introduction to the Issue Policy Recommendations for Corporate Social Responsibility for Arctic Petroleum», realizaron un análisis sobre la RSE en la explotación y extracción del petróleo, enfocándose con especial atención al impacto que genera al medio ambiente.

La metodología aplicada por Poussenkova et al. (2016), fue cuantitativa, por medio de un trabajo de campo en cuatro estudios de casos en los que se realizaron entrevistas semiestructuradas: Hammerfest, Murmansk, Komi Republic y Nenets Autonomous Okrug (NAO). Las unidades de información fueron miembros de la población local, autoridades regionales y locales, ONG y representantes de compañías petroleras. Los hallazgos mas sobresalientes se relacionan con el desarrollo de un modelo de insumo-producto de análisis económico para determinar cuánto petróleo contribuye al mejoramiento de la economía en la República de Komi (Federación de Rusia). Los resultados se compararon con Noruega con el fin de investigar la capacidad de la industria petrolera para impulsar el crecimiento regional en ambos países (Rusia y Noruega).

Por otra parte, Tai \& Chuang (2014), en «Corporate Social Responsibility» hacen referencia a que las empresas deberían, no sólo ser una provechosa herramienta para el crecimiento de las ciudades, sino también responsables en el cuidado de los grupos de interés (stakeholders) y sus necesidades. De este modo se vislumbra que las prácticas empresariales requieren una estructura que cumpla con los estándares y los objetivos para el desarrollo sostenible.

La metodología utilizada por Tai \& Chuang (2014) fue cuanti-cualitativa. Los hallazgos que sobresalen en la investigación especifican que las multinacionales son 
responsables de los grupos de interés, por tanto, las empresas deben centrarse en los valores de la economía global y de la responsabilidad social que crea objetivos a largo plazo de seguridad para la sucesión de transacciones por parte de las necesidades de todos los proveedores, inversores y empleados.

Tai \& Chuang (2014), concluyen a favor de la responsabilidad social y promueven esta estrategia desde la teoría conceptual hasta la práctica, como una de las más importantes en la industria. Se sugiere en la precitada investigación que es menester realizar mayor inversión en marketing para tener fidelización de clientes, por medio de la innovación de los bienes y servicios.

Otro aporte de interés resulta de la investigación desarrollada por Cheng, Ioannou \& Serafeim (2014), titulada «Corporate social responsibility and access to finance», en la que se resalta que el desempeño superior en las estrategias RSE conduce a un mejor acceso a la financiación de las empresas. Dicha investigación se corresponde con una metodología cuantitativa en la que se plantea y comprueba la hipótesis de que un mejor acceso a las finanzas puede atribuirse a la reducción de los costos de agencia debido al mayor compromiso de las partes interesadas y a la reducción de la asimetría informacional debido al aumento de la transparencia.

La hipótesis planteada por Cheng, Ioannou \& Serafeim (2014), fue probada mediante un estudio a 45 empresas, resultando que las empresas con un mejor desempeño de RSE enfrentan restricciones de capital significativamente menores. En los hallazgos se proporcionan evidencias de que tanto el mejor compromiso de las partes interesadas, como la transparencia en torno al desempeño de la RSE, son importantes para reducir las limitaciones de capital. Los resultados se confirman con varias medidas alternativas de restricciones de capital, ejecutando un análisis basado en las calificaciones para el desempeño de la RSE, con un enfoque de variables instrumentales y de ecuaciones simultáneas.

También es menester señalar el estudio de Ospina y Sotelo «Responsabilidad Social Empresarial: Beneficios económicos, sociales y ambientales para los stakeholders» (2013), en el que plantean como objetivo establecer la situación de las empresas del sector construcción frente al tema de las RSE como factor generador de valor económico a partir de información ya documentada de algunos modelos propuestos por diferentes autores y casos de algunas empresas del sector. El abordaje metodológico del estudio de Ospina y Sotelo (2013) consistió en realizar un análisis a los informes sociales o de sostenibilidad, en primera instancia, pero cuando las empresas no presentaban este informe acudieron a los informes de gestión de cada empresa. Por medio de estos informes y el sistema de variables, se desarrolló una investigación cualitativa y descriptiva.

Ospina y Sotelo (2013), manifestaron que la importancia de la RSE no radica solo en beneficiar a los grupos de interés o stakeholders, pues las organizaciones deben aspirar a mantener un equilibrio social, de modo que puedan aminorarse los daños ocasionados a la sociedad. Concluyen que los grupos de interés pueden obtener beneficios económicos, sociales y ambientales cuando las empresas son socialmente responsables, puesto que estas últimas tienen el objetivo de generar riqueza para quienes hacen parte o poseen una relación directa; en este sentido la labor es intrínsecamente dependiente de la sociedad y su progreso. 
Como aporte, el trabajo de Ospina y Sotelo (2013) brinda información al lector acerca de cómo la Responsabilidad Social Empresarial es un factor útil y eficaz para mejorar la productividad de las empresas de Medellín (Colombia) ya que ésta satisface las necesidades de un individuo o grupo de individuos que pueden afectar o ser afectados por el logro de los objetivos de una organización. A su vez verifican que la RSE constituye una gran oportunidad para mejorar los beneficios de las empresas.

\subsection{Bases legales de la Responsabilidad Social Empresarial en Colombia}

En cuanto al sustento legal, la Constitución Política de 1991 de Colombia menciona la responsabilidad social en los artículos 20, 73, 95 y 333, enfocados principalmente en la responsabilidad de los ciudadanos. Sin embargo, el artículo 333, reconoce la actividad empresarial como una fuente para el desarrollo social del país desde la perspectiva de su función social. Otro instrumento legal lo representa la sentencia T-247 de 2010, en la cual se menciona que en compensación al beneficio lucrativo que reciben las empresas, éstas deben asumir un compromiso social. No obstante, esta decisión no es de obligatorio cumplimiento para las empresas, ya que busca que sea más de forma voluntaria y no impositiva (Sentencia T-247, 2010).

Igualmente en Colombia, se han tramitado cuatro proyectos de ley sobre el tema: Proyecto Ley 153 (2006) y Proyecto Ley 031 (2007), ambos sobre la responsabilidad social y el medio ambiente, mientras que el Proyecto Ley 058 (2009) y Proyecto Ley 070 (2010), versan sobre RSE y protección infantil.

\subsection{Responsabilidad Social Empresarial}

La Responsabilidad Social Empresarial (RSE), hace referencia al rol que tiene la empresa con la sociedad y basa sus actividades en principios éticos conforme a la Ley. Se refiere a la relación que la empresa tiene con la sociedad, con su entorno y que se materializa en la implementación de acciones de beneficio mutuo (Duque, Cardona \& Rendón, 2013).

Peláez \& García (2014), definen la RSE como un enfoque estratégico que hace parte de la gestión de la organización, aportando ventajas competitivas y diferenciación de las empresas frente a sus grupos de interés (stakeholders), la sociedad y el entorno.

Para la Comisión Europea (2001), la RSE es comprendida como la integración voluntaria por parte de las empresas para trabajar en pro de problemas sociales y ambientales en concordancia con sus operaciones comerciales y su relación con la sociedad. Además, señala que ser socialmente responsable no implica únicamente cumplir con las obligaciones jurídicas, sino también ir más allá de su cumplimiento, invirtiendo más en capital humano, en el entorno y en las relaciones con los grupos de interés. Con base en esta definición, la RSE se fundamenta en tres aspectos: a) La voluntad, b) Identidad y sostenibilidad y, c) Relación con los grupos de interés.

En ninguno de estos aspectos se plantea el cumplimiento de las normas como un factor inherente a la RSE, constituyendo éstas tan sólo algunas de las acciones que deben implementar las organizaciones. En esta línea, las acciones de responsabilidad social no deben girar en torno al cumplimiento de una obligación normativa, sino que deben ser parte del entendimiento estratégico del relacionamiento de la organización. La voluntad debe partir del interés particular que tiene la empresa por emprender 
acciones en pro del entorno social y ambiental en el que está inmersa. El principio de identidad y sostenibilidad indica el compromiso que tiene la empresa con alinear su crecimiento económico con la sostenibilidad ambiental, lo que se denomina «desarrollo sostenible». En este sentido, la RSE se enfoca en el bienestar de todos sus grupos de interés: clientes, accionistas, proveedores, empleados; como parte integral de la misma.

Tal y como lo plantea la Secretaría General del Gobierno de Salta (2014), una de las mayores cualidades que deben desarrollar las organizaciones en la actualidad es lograr consolidar todos los aspectos que permitan catalogarla como «socialmente responsable». El desarrollo económico debe estar alineado con el compromiso social y el respeto al medio ambiente, de tal forma que se logren satisfacer las necesidades inmediatas sin sacrificar las futuras. De esta manera se contribuye también al desarrollo sostenible y se revierte en la sociedad parte del beneficio que la misma origina a la organización, al sistema productivo y por consiguiente, a la economía.

Por su parte, Rincón, Caridad \& Salazar (2017), señalan que «la responsabilidad social es una función en la gestión y desarrollo de las organizaciones, amparada en el estado de consciencia colectivo, para el impulso de acciones de sensibilidad social, económica y medioambiental» (p. 48).

El concepto de RSE indudablemente ha presentado una evolución. Inicialmente se entendía como una responsabilidad básica de ayuda a la sociedad, y en la línea de tiempo ha logrado abarcar las áreas de influencia de su actuar (lo social, lo ambiental y lo económico), causando una presencia activa dentro de la sociedad y una actividad que se halla dentro de la estrategia corporativa de las organizaciones. Al respecto De la Garza (2013), afirma:

Cada vez más las empresas perciben que la responsabilidad social es un tema que no está restricto solamente a las acciones sociales o ambientales desarrolladas por la organización en la comunidad, sino que implica también las prácticas de diálogo e interacción con los diversos públicos de la empresa... Para que la empresa trabaje en el tema de la responsabilidad social, en una perspectiva sistémica y amplia, es necesario que éste sea incorporado a los procesos de gestión y, por lo tanto, tratarlo como parte de las estrategias de negocio y del sistema de planeación interna de la empresa (p. 23).

Según Carroll (1991) la orientación en la aplicación de la RSE en las organizaciones se presenta en cuatro dimensiones, que integran la mayoría de los segmentos del sector de construcción. Éstas son: la dimensión económica, legal, ética, filantrópica y medioambiental.

- La dimensión de responsabilidad económica: Espera que las empresas generen lucro, su naturaleza es efectivamente económica y es tomada como el beneficio que producen las empresas al vender bienes y servicios para el funcionamiento del capitalismo.

- La dimensión de responsabilidad legal: Muestra que las empresas obedecen a una naturaleza totalmente representada en un contexto jurídico, su responsabilidad será ir en concordancia con los reglamentos y regulaciones del ramo. 
- La dimensión de responsabilidad ética: Indica que las empresas deben demostrar sus deberes y valores con la naturaleza de comportamiento hacia la ética, sin perjudicar a sus grupos de interés.

- La dimensión de responsabilidad filantrópica: Demuestra que la sociedad o empresa debe ser solidaria, enfocada a la ayuda hacia los demás como una gestión social cada vez más efectiva.

La RSE puede ser categorizada en atención a diversos factores o indicadores, considerando el «triple bottom line»-explicado ut supra-, herramientas e instrumentos. Un ejemplo de esto son los «Objetivos de Desarrollo del Milenio» ${ }^{1}$, y muy particularmente las dimensiones de obligación social, reacción social y sensibilidad social de Donnelly, Gibson, Ivancevich \& Konopaske (2011).

Lo anterior permite vislumbrar cómo la sociedad en general y los grupos de interés asociados a la dinámica comercial, en particular, resultan cada vez más conscientes de la importancia de los aspectos sociales, económicos y medioambientales, reflejados en acciones de cuidado del medioambiente, de su papel como consumidor y como usuario. Asimismo, las empresas están preocupadas por satisfacer las demandas de los públicos y en atender aspectos no solo intrínsecos al proceso organizacional sino que impacten positivamente en el entorno, y, sobre todo, favorezcan a los públicos organizacionales; entendiendo que la principal responsabilidad de una empresa está en ser productiva, mantenerse activa; solo así pudiendo garantizar la operatividad y permanencia en el mercado.

Con las estrategias de RSE se pretende cumplir con los requerimientos de los diversos grupos de interés de la organización (stakeholders), teniendo como punto de referencia los pilares de la RSE que son el compromiso social, económico y medioambiental. Mediante la gestión integral de estas áreas se producen mejoras en las organizaciones, al imponer mecanismos direccionados a prácticas responsables que integren las estrategias corporativas impulsadas por los sistemas de gestión. Según Yepes (2007) estas estrategias son: a) Mercado base de pirámide, b) cadena de abastecimiento, d) Clúster de Responsabilidad Social Empresarial y, d) Marketing Social Responsable.

\subsection{Herramientas de Responsabilidad Social Empresarial}

En concordancia con lo anterior, la adopción de la responsabilidad social por parte de las organizaciones se ha realizado a partir de diversas herramientas. En tal sentido en abril de 1991, la Organización de Naciones Unidas y la Comisión Económica para América Latina y el Caribe (CEPAL) presentaron el documento «Nuestra Propia Agenda» en el que se rescatan los siguientes trece aspectos:

a) Paz y desarme mundial.

b) Nuevas relaciones económicas.

1 Disponible en: https://goo.gl/sxgQtE (23/07/2018). 
c) Enfrentamiento a la pobreza.

d) Políticas económicas inconvenientes al desarrollo sustentable de la región.

e) Aprovechamiento de recursos naturales.

f) Tratamiento de los problemas ambientales globales.

g) Nuevos mecanismos de financiamiento para la ejecución de programas de conservación ambiental.

h) Ámbitos y alcances de cooperación técnica.

i) Condicionalidades en materia ambiental.

j) Cooperación financiera requerida para adelantar la estrategia de desarrollo sustentable.

k) Cambio de los patrones de consumo en el norte.

1) El tratamiento del tema de las drogas.

m) Población.

Más recientemente el «Pacto Global», anunciado en el Foro de Davos en 1999, conforma un instrumento estratégico de libre adhesión, a partir de 10 principios que actúan en cuatro áreas consideradas de interés en el ámbito de la responsabilidad social y el desarrollo sustentable: Derechos Humanos, ámbito laboral, medioambiente y lucha contra la corrupción.

En 2000, 189 países miembros de las Naciones Unidas proponen los Objetivos del Desarrollo del Milenio, los cuales debían ser alcanzados para el 2015; estos objetivos son:

a) Erradicar la pobreza extrema y el hambre.

b) Lograr la enseñanza primaria universal.

c) Promover la igualdad entre los géneros y la autonomía de la mujer.

d) Reducir la mortalidad infantil.

e) Mejorar la salud humana.

f) Combatir el VIH/SIDA, el paludismo y otras enfermedades.

g) Garantizar la sostenibilidad del medioambiente.

h) Fomentar una asociación mundial para el desarrollo.

No obstante, en 2015 los progresos de estos objetivos fueron evaluados, dando paso a una reformulación que se extienden en la actualidad a 17 Objetivos de Desarrollo Sostenible $(\mathrm{ODS})^{2}$, basados en los progresos alcanzados a través de los Objetivos de Desarrollo del Milenio (ODM). Esta adaptación se hizo patente en la formulación de la denominada «Agenda 2030».

A la fecha, en Colombia se sigue trabajando de manera intensiva en la implementación de estrategias que posibiliten la aceleración del logro de los ODM, identificando «cuellos de botella» e implementando soluciones de corto y mediano plazo para garantizar el cumplimiento de las metas más rezagadas, apoyando la construcción de políticas públicas que permitan la superación de la pobreza extrema y el hambre, con

2 Disponible en: https://goo.gl/jKJhA9 (23/07/2018). 
esfuerzos realizados desde instituciones del Gobierno Nacional como las lideradas por la Agencia para la Superación de la Pobreza Extrema (ANSPE). En este mismo propósito vienen trabajando los distintos Fondos, Programas y Agencias del Sistema de las Naciones Unidas en el país, implementando soluciones en temas de hábitat, educación, salud, género, gobernabilidad, desarrollo económico incluyente y generación de una cultura de paz (ONU, 2016).

En este orden de ideas y desde el compromiso político del país, el Plan Nacional de Desarrollo 2014-2018 «Todos por un nuevo país» contempla objetivos y metas estratégicas orientadas a la generación y fortalecimiento de las condiciones necesarias para construir una sociedad en paz, equitativa y educada. Del mismo modo, y con el propósito de prepararse para la implementación de los ODS, el 18 de febrero de 2015, Colombia dio un paso que lo puso a la vanguardia del tema en el mundo, mediante la creación de la «Comisión Interinstitucional de Alto Nivel para el Alistamiento y la Efectiva implementación de la Agenda de Desarrollo Post 2015 y sus ODS» (ONU, 2016).

La tarea actual consiste en la adopción de los ODS al contexto colombiano de la manera más pronta y efectiva posible, a través del diseño e implementación de políticas públicas y la incorporación dentro de los planes de Gobierno locales (dentro de los nuevos períodos de gobierno), que soporten el logro de estas nuevas metas. (ONU, 2016, p.20).

Asimismo existen otras herramientas que fijan las áreas prioritarias de atención para la Responsabilidad Social Empresarial (RSE), tales como:

- $\quad$ Global Reporting Initiative (GRI).

- AccountAbility 1000 (AA1000).

- Gestión Ética y Gobierno Corporativo (Norma SGE21:2008) (Forética, 2017).

- Libro Verde de la Unión Europea (Comisión Europea, 2001).

- Gestión frente a los colaboradores (SA 8000) (Departamento Económico y Social, 2015).

- $\quad$ ISO 26000 (ISO 26000, s.f.).

- Gestión Medioambiental (ISO 14001) (ISO 14001, 2015).

- Gestión de la Calidad (ISO 9000) (ISO 9000, 2015).

- Indicadores Ethos.

\section{Materiales y método}

La presente investigación se realiza con un enfoque de tipo integrador u holístico, el cual permite al investigador comprender las distintas fases que conlleva una investigación, facilita conocer lo que ya se ha descrito, genera aportes a lo ya dicho previamente y, además, acepta la integración de métodos y técnicas. Hurtado (2012), con la propuesta del ciclo holístico, presenta una síntesis integradora del proceso investigativo lo que impulsa una visión completa del objeto de estudio, permitiéndole al investigador ser más que un observador en la investigación y participar de forma activa en la re-construcción de los conocimientos. En esta línea se realizó una investigación de tipo 
descriptiva para abordar las estrategias de Responsabilidad Social Empresarial (RSE) en las empresas del sector de la construcción de la ciudad de Medellín (Colombia).

Se partió de una revisión documental para la identificación y selección de la información que llevó a la conceptualización del tema tratado, revisando las teorías y definiciones existentes, para así proceder a su comparación, valoración e integración en la búsqueda de las particularidades que ayuden en su categorización. En el desarrollo de la investigación se cumplió con las fases predictiva, proyectiva, interactiva, confirmatoria y evaluativa, tal y como lo plantea Hurtado (2012).

La población de estudio estuvo conformada por las empresas del sector construcción de la ciudad de Medellín, tomándose como referente muestral las inscritas en FENALCO Antioquia, siendo un total de 13 empresas, todas legalmente constituidas. En este sentido, se aplicó la técnica del censo poblacional, considerando que la muestra en estudio es reflejo de la totalidad de la población. La técnica utilizada fue la encuesta a través de un cuestionario conformado por 15 ítems de preguntas cerradas.

\section{Análisis de resultados}

Con el objetivo de describir las estrategias de Responsabilidad Social Empresarial empleadas por las organizaciones del sector construcción en Medellín, se presentan, a continuación, los resultados obtenidos:

Figura 1. Orientación de la estrategia

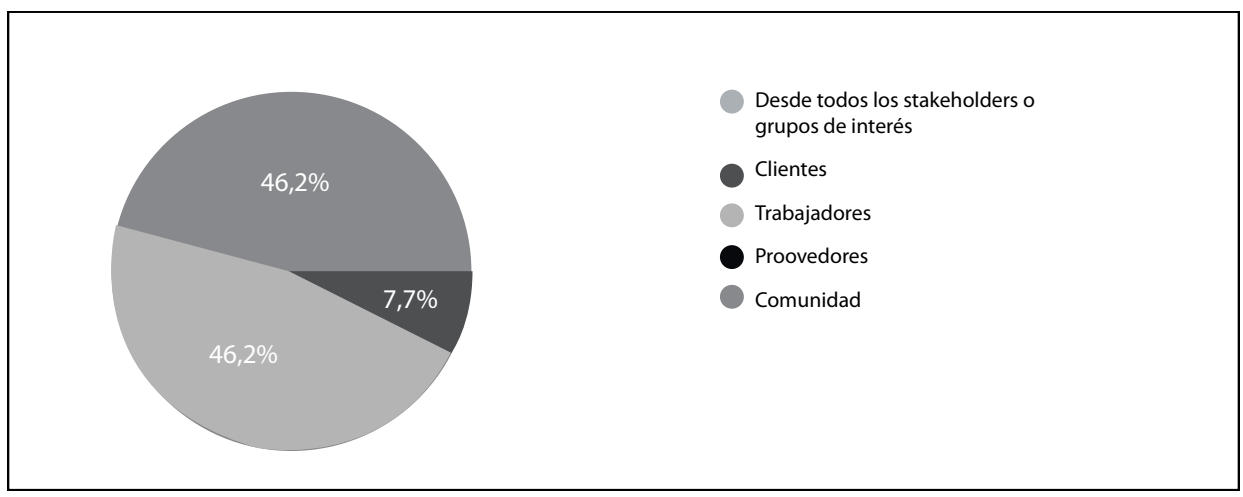

Como puede observarse en la figura 1, el 46,2\% de las empresas manifiesta que sus estrategias de Responsabilidad Social Empresarial (RSE) están orientadas a los trabajadores y en misma proporción hacia la comunidad, en tanto solo un 7,7\% a los clientes. De esto se puede interpretar que la orientación de la RSE está alineada hacia la dimensión operativa de la empresa, concentrada en los colaboradores internos y proveedores. 
Figura 2. Tipo de estrategia

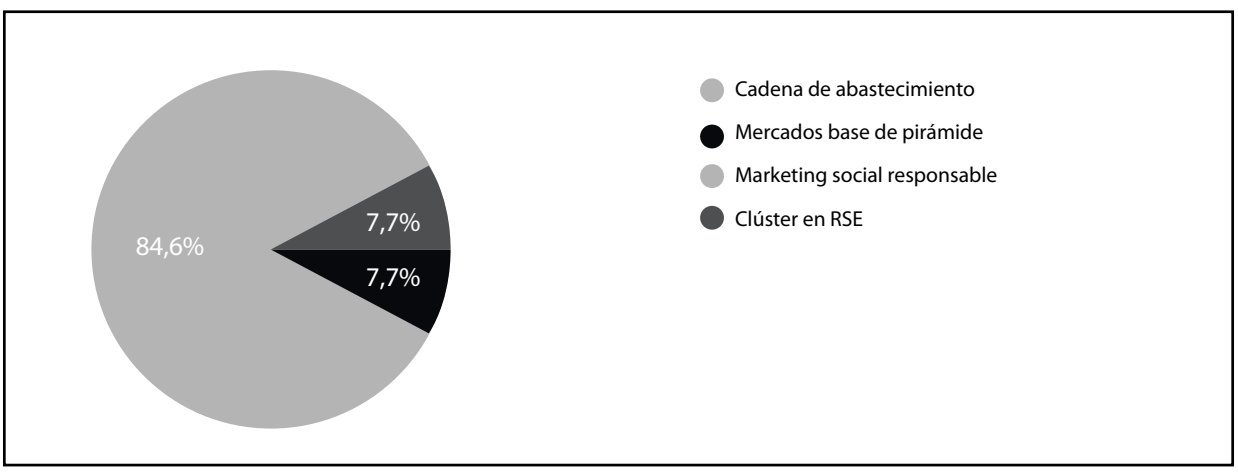

La figura 2 muestra cómo el 84,6\% de las empresas aplica como estrategia de Responsabilidad Social Empresarial el marketing social responsable; seguido del 7,7\% que aplica mercados base de pirámide y el restante 7,7\% mediante clúster en RSE. Llama la atención que en tanto la orientación de la RSE está en torno a los públicos internos e intermedios, la estrategia empleada esté focalizada en el marketing social responsable, lo que podría interpretarse como una mirada hacia los públicos externos o el entorno organizacional; al tiempo que podría vislumbrarse como una estrategia genérica. A este respecto se pudo observar que el 84,6\% de las empresas ha aplicado estrategias de RSE entre 1 a 5 años, 7,7\% menos de 1 año, y el restante 7,7\% entre 5 a 10 años, lo que señala que el sector está atento a la necesidad de aplicar estrategias de responsabilidad social.

Figura 3. Dimensiones de aplicación de la estrategia

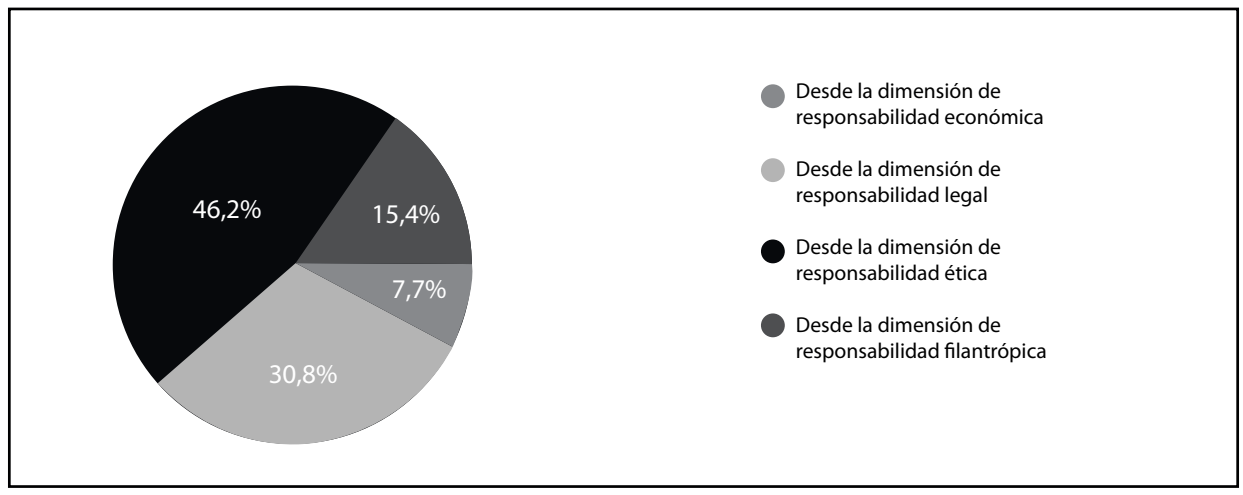

En lo concerniente a las dimensiones de aplicación (figura 3), las estrategias de RSE de las empresas del sector construcción de Medellín se orienta en un 46,2\% a la responsabilidad ética; 30,8\% considera la aplicación desde la dimensión legal; el $15,4 \%$ se enfoca en la dimensión filantrópica, y solo un 7,7\% se centra en la responsabilidad económica. 
Figura 4. Tipos de herramientas de RSE que aplica la empresa

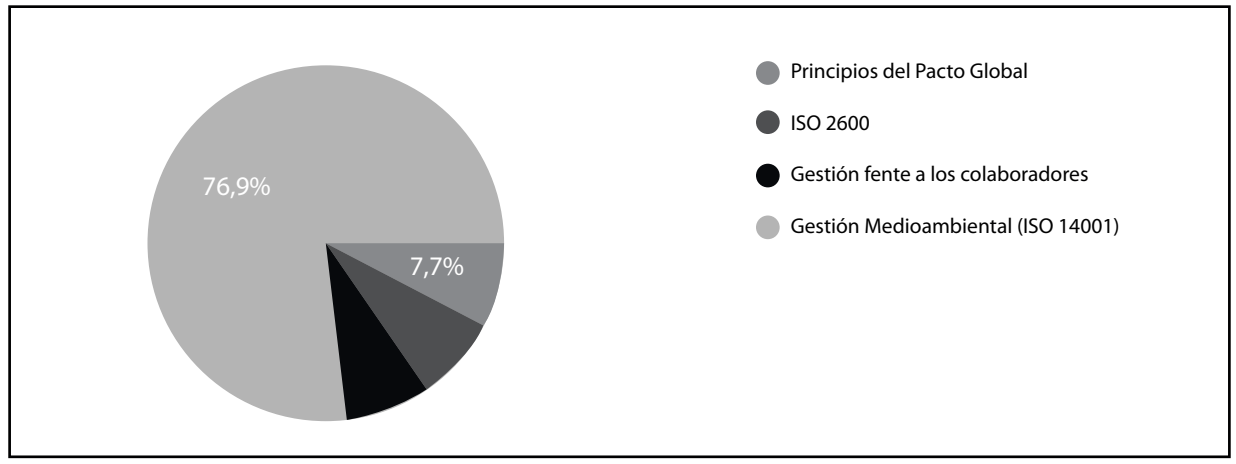

Al considerar los tipos de herramientas de RSE que aplican las empresas ut supra referenciadas (figura 4), se puede observar que el 76,9\% aplica como herramienta de RSE la norma ISO 14001 sobre Gestión Medioambiental, 7,7\% aplica principios del Pacto Global, otro 7,7\% aplica la norma ISO 2600, coincidentemente 7,7\% opta por gestión frente a los colaboradores. Se evidencia que estas empresas se encuentran altamente influenciadas por la norma ISO 14001 para determinar las estrategias de RSE que aplican.

Figura 5. La empresa posee herramientas para conocer la mejora de los procesos internos y externos sobre RSE

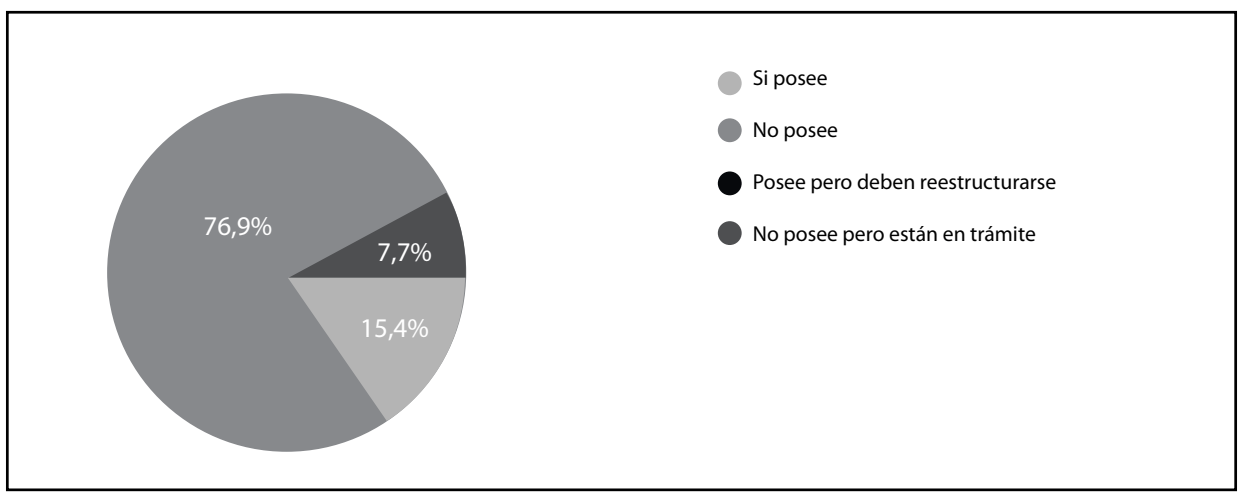

Como se observa en la figura 5, el 76,9\% de las empresas no posee un programa o herramienta para conocer la mejora en los procesos internos y externos sobre RSE; $15,4 \%$ cuenta con mecanismos de evaluación y control; mientras que $7,7 \%$ no cuenta con ellos, pero aseguran que están en trámite. Una vez más se confirma que la mayoría de las empresas se están preocupando por la estructuración de estrategias de RSE y por su implementación, pero pocas llevan un control sobre las mismas y/o determinan su impacto. 
Figura 6. Presentación de Informes

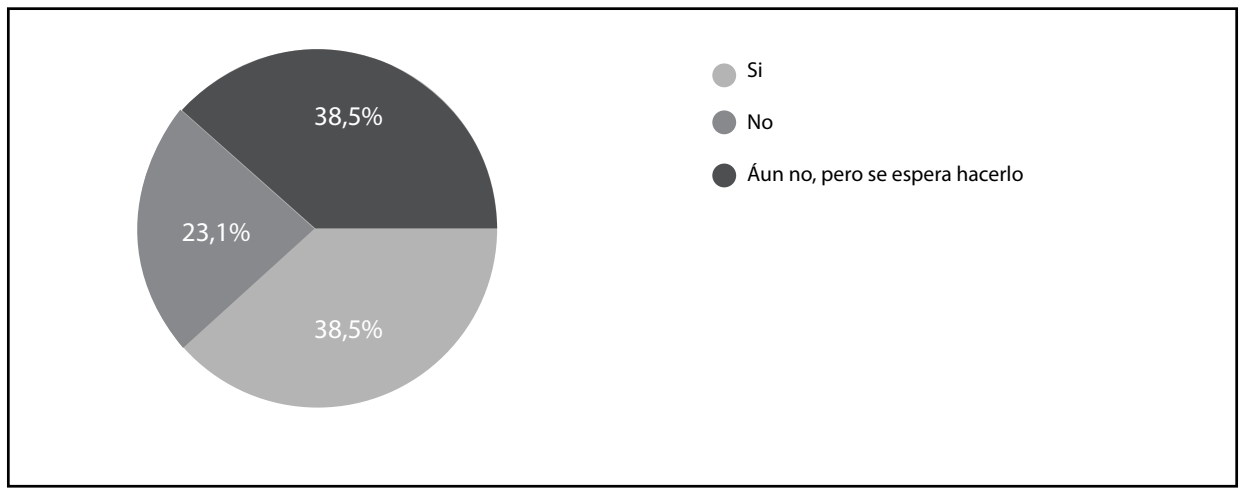

Al considerar la presentación de informes en torno a la RSE (figura 6), el 38,5\% de las empresas señala que sí los realiza, reportando impactos positivos y negativos; esto frente a una proporción igual de empresas que aún no lo hacen, pero esperan hacerlo; mientras que $23,1 \%$ no lo hace.

Estos resultados demuestran que hace falta que las empresas se enfoquen en mayor medida en la evaluación de impactos; en establecer mecanismos de control, monitoreo, evaluación y retroalimentación y así garantizar que las estrategias cumplan con los objetivos para las cuales fueron diseñadas.

\section{Discusión y análisis}

Tras el análisis descriptivo de los datos obtenidos se pudo contrastar la información con los postulados teóricos. De tal manera, al considerar el primer objetivo en el que se propuso identificar la orientación estratégica de la Responsabilidad Social Empresarial con los públicos organizacionales, se pudo evidenciar efectivamente en la mayoría de la muestra de análisis una orientación de la estrategia hacia a los públicos organizacionales internos e intermedios, con lo cual se ratifican las consideraciones de Rincón (2017, párr.2) quien señala:

Si bien para muchos está de moda, orienta la capacidad de responder mediante la articulación de acciones coordinadas a las necesidades de un colectivo cada vez mejor informado, más organizado y dispuesto a lograr metas y objetivos comunes.

Con respecto al segundo objetivo, que consiste en identificar las estrategias de responsabilidad social aplicadas por las empresas del sector construcción en Medellín, se pudo precisar que la mayor parte de las empresas se enfocan en estrategias orientadas a la comunidad y a los trabajadores, siendo las estrategias de mayor aplicación el marketing social responsable, en correspondencia con lo señalado por Yepes (2007).

El tercer objetivo, que consiste en determinar las herramientas de responsabilidad social empleadas por las empresas del sector construcción en Medellín, se pudo vislumbrar que la mayoría de empresas aplica como herramienta de RSE la norma ISO 14001 sobre Gestión Medioambiental. Precisamente, esta norma apunta a que 
cada empresa diseñe e implemente un Sistema de Gestión Ambiental mediante el cual logre un equilibrio entre el medio ambiente, la sociedad y la economía. Pese a que es la norma de mayor aplicación entre estas empresas, se identificó que la mayoría no posee ningún programa o herramienta para conocer la mejora de los procesos internos y externos sobre RSE; aspecto que debería estar solucionado al implementar un Sistema de Gestión Ambiental, lo cual evidencia que es necesario evaluar y mejorar la aplicación de la norma.

\section{Conclusiones}

La Responsabilidad Social Empresarial influencia aspectos empresariales como calidad, productividad, competitividad, identidad, imagen y reputación. Asimismo, la RSE se rescata como una dimensión interna de las empresas, la dinámica organizacional y por ende abocada a los colaboradores internos y grupos de interés con estrecha vinculación al nivel operativo organizacional, lo que establece un reflejo en la cultura y el clima organizacional; al tiempo que a nivel de los grupos de interés externos a la organización, perciben los aspectos de responsabilidad mediante las dinámicas de prestación de servicio y comercialización de la empresa, lo que a menudo genera posicionamiento, fidelización y generación de valor de la marca empresarial y sus marcas asociadas. Por tanto se hace necesario realizar un mayor esfuerzo al orientar las estrategias de RSE en torno a los públicos organizacionales y grupos de interés de manera equilibrada. Se recomienda en este sentido crear un mapa de públicos y grupos de interés en torno a las empresas del sector construcción.

Asimismo, se recomienda la orientación de la estrategia de responsabilidad social de manera equilibrada en atención a los diversos factores o indicadores considerando tanto al «triple bottom line», como a las herramientas e instrumentos, tanto los mencionados como ODM, los ODS y las dimensiones de obligación social, reacción social y sensibilidad social que presentan Donnelly, Gibson, Ivancevich \& Konopaske (2011).

Si bien la mayoría de las empresas del sector construcción en la ciudad de Medellín (Colombia) implementan estrategias de RSE y tienen clara su orientación y herramientas de aplicación, se evidencia la necesidad de mejorar en cuanto a mecanismos de evaluación y seguimiento sobre la efectividad de dichas estrategias; puesto que se ha identificado que la mayoría de empresas se están quedando en la planeación e implementación, y pocas han pasado a la evaluación y retroalimentación para lograr un verdadero impacto.

La Norma ISO 14001, en la cual se establecen pautas para el diseño e implementación de un Sistema de Gestión Ambiental en las organizaciones, sirve de orientación para la mayoría de empresas del sector construcción en Medellín. En esta línea, se recomienda la diversificación de estrategias de responsabilidad social en correspondencia con los factores e indicadores que proveen las diversas herramientas y mecanismos de responsabilidad social empresarial referenciadas en precedencia.

La práctica de la Responsabilidad Social Empresarial, en la mayor parte de las empresas del sector construcción en Medellín es muy joven, teniendo menos de diez años de ejecución, y se enfoca en estrategias orientadas a la comunidad y a los trabajadores, principalmente. En este sentido aplican estrategias de mercadeo socialmente 
responsable y usan fundamentalmente herramientas del medioambiente, con lo que se vislumbra como una estrategia de responsabilidad social empresarial incipiente.

\section{Apoyos y agradecimientos}

Artículo derivado del proyecto de investigación para optar a grado titulado Responsabilidad Social: Factor generador de valor en empresas de la construcción en Medellín, en el marco del Proyecto de Investigación: Comunicación Organizacional: Función Articuladora de las Organizaciones con sus Públicos, en la Línea Gestión y Estudios de las Organizaciones del Grupo RED en el Tecnológico de Antioquia, Institución Universitaria.

\section{Referencias}

Cardona, A. (2017) La historia de casi 100 años de la responsabilidad social empresarial. La República. (https://goo.gl/GqngTS).

Carroll, A. (1991). The pyramid of corporate social responsibility: Toward the moral management of organizational stakeholders. Business Horizons, 34(4), 39-48. https://doi.or$\mathrm{g} / 10.1016 / 0007-6813(91) 90005-\mathrm{G}$

Cheng, Ioannou \& Serafeim. (2014). Corporate social responsibility and access to finance. Strategic Management Journal, 35(1), 1-23. https://doi.org/10.1002/smj.2131

Comisión Europea (2001). Libro Verde: Fomentar un marco europeo para la responsabilidad social de las empresas. Bruselas: Comisión Europea. (https://goo.gl/XPA9jK).

Congreso de Colombia (06 de agosto de 2009). Por la cual se definen normas sobre la responsabilidad social empresarial, la protección infantil y se dictan otras disposiciones. [Proyecto de Ley 070] (https://goo.gl/1M7Krn).

Congreso de Colombia (26 de julio de 2007). Por la cual se definen normas sobre la Responsabilidad Social y medioambiental de las empresas y se dictan otras disposiciones. [Proyecto de ley 031] (https://goo.gl/zwB45E).

Congreso de Colombia (27 de noviembre de 2006). Por la cual se reglamenta la Responsabilidad Social de las empresas, y se dictan otras disposiciones.[Proyecto de ley 143] (https://goo. $\mathrm{gl} / \mathrm{k} 6 \mathrm{LeqH})$.

Constitución Política de Colombia [Const.] (1991). Bogotá.

Corte Constitucional de Colombia. Sentencia T-247. Magistrado Ponente Humberto Sierra Porto. 2010.

DANE (2017). Boletín técnico sobre RSE. (https://goo.gl/WtfHnn).

De la Garza, O. (2013). Responsabilidad Social Empresarial en México ¿Costo o beneficio? (Tesis de maestría). Universidad Autónoma de Nuevo León. San Nicolás de los Garza, Nuevo León.

Departamento Económico y Social (2015). Certificación SAO 8000. (https://goo.gl/nSJkwh).

Donnelly, J., Gibson, J., Ivancevich, J. \& Konopaske, R. (2011) Organizaciones: comportamiento, estructura y procesos. México: McGrawHill.

Duque, Y., Cardona, M., \& Rendón, J. (2013). Responsabilidad Social Empresarial: Teorías, índices, estándares y certificaciones. Cuadernos de Administración, 29(50) (https://goo.gl/rhH3ri).

Forética (2017). Sistema de Gestión Ética y Socialmente Responsable. (https://goo.gl/SNrYg4).

Global Reporting Initiative (2011). Cómo usar las directrices del GRI, en conjunto con la norma ISO 26000. (https://goo.gl/mt5jFV).

Hurtado, J. (2012). Metodología de la Investigación. Guía para la comprensión holística de la ciencia. Bogotá: Quirón Ediciones.

ISO 14001 (2015). (https://goo.gl/7bHhJ7)

ISO 26000 (s.f.). Guía de Responsabilidad Social. (https://goo.gl/6ywdVw). 
ISO 9000 (2015). Sistema de Gestión de la calidad. (https://goo.gl/U7Xcqj).

Ospina, E., \& Sotelo, M. (2013). Responsabilidad social empresarial: beneficios económicos, sociales y ambientales para los stakeholders (Trabajo de grado). Medellín: Universidad de Antioquia. (https:// goo.gl/xzwj2n).

Padilla, C. P., Arévalo, D., Bustamante, M. A., \& Vidal, C.L. (2017). Responsabilidad Social Empresarial y desempeño financiero en la industria del plástico en ecuador. Información tecnológica, 28(4), 93-102. https://doi.org/10.4067/S0718-07642017000400012

Peláez, J., \& García, M. (2014). Responsabilidad social empresarial y gestión humana: una relación estratégica aplicada desde un modelo explicativo. Entramado 10(2). 90-111. (https:// goo.gl/2za2iQ).

Pérez, G. J. (2015). La infraestructura del transporte vial y la movilización de carga en Colombia. Documentos de Trabajo sobre Economía Regional y Urbana 64. pp.73 Banco de la República. CEER. Cartagena. Colombia. (https://goo.gl/h8whw8).

Poussenkova, N., Nikitina, Wilson Rowe, E. \& Fjaertoft, D. (2016). Corporate social responsibility. Russian Analytical Digest, 181(1), 3-7. (https://goo.gl/XF8GP3).

Rincón, Y. (2017). Relaciones públicas socialmente responsables [entrada de blog] (https://goo. $\mathrm{gl} / \mathrm{F} 1 \mathrm{CrzS})$.

Rincón, Y., Caridad, M., \& Salazar, C. (2017). Responsabilidad social en universidades de gestión privada en Barranquilla. Revista Ciencias Sociales, 23(3), 48-62. (https://goo.gl/PN1tz6).

Rincón, Y. \& Ramírez, R. (2018). Comunicación responsable en las organizaciones. En Martha Lucía Quintero Garzón \& María Dolores Sánchez Fernández (Comp.), Responsabilidad Social Corporativa. Una mirada integral en América Latina (pp. 93-100). Cali, Colombia: Editorial Universidad del Valle. Recuperado de https://goo.gl/fn93kF. ISBN: 978-958-765-572-8

Secretaría General del Gobierno de Salta (2014). Conceptos generales sobre la responsabilidad social corporativa. Gobierno de la provincia de Salta. Chile. (https://goo.gl/RgjGKC).

Tai, F.-M., \& Chuang, S.-H. (2014). Corporate social responsibility. Ibusiness, 6(03), 117. https://doi. org/10.4236/ib.2014.63013.

Yepes, G. (2007). Responsabilidad Social Empresarial fundamentos y aplicaciones en las organizaciones de hoy. Bogotá: Universidad Externado de Colombia. 